\title{
(Committee of the Executive Committee)
}

President: F. B. Wood

Vice President: E. A. Muller

Organizing Committee (Comite d'Organisation): A. A. Boyarchuk, J. Delhaye, D. A. MacRae, P. H. Routly, J. Sahade, F. G. Smith, C. R. Tolbert, H. H. Voight, S. H. Ye

While the chief function and methods of operation of the Commission have remained much the same as in the past, there has been a gradual evolution in the nature of the proposals submitted. A greater fraction now come from countries in which the study of astronomy on a professional basis is as yet very recent and more proposals are being received from relatively young astronomers, although admittedly the distinction between a "young" and an "established" astronomer is not always easy to make. The commission may wish to consider whether or not it is advisable to reconsider its guidelines. Grants awarded during the interval 30 November 1981 and 31 January 1985 were the following.

$\begin{array}{lll}\text { Grantee } & \text { Origin } & \text { Destination } \\ \text { C. E. Akujor } & \text { Nigeria } & \text { India/France } \\ \text { P. Chen } & \text { China } & \text { USA } \\ \text { E. Corbelli } & \text { Italy } & \text { USA } \\ \text { M. K. Das } & \text { India } & \text { England } \\ \text { V. N. Dermendjiev } & \text { Bulgaria } & \text { France } \\ \text { S. K. Jain } & \text { India } & \text { England } \\ \text { L. Jing } & \text { China } & \text { Scotland } \\ \text { M. Kaisig } & \text { Germany } & \text { USA } \\ \text { S. Manorama } & \text { India } & \text { Italy } \\ \text { M. V. Mekkanden } & \text { India } & \text { Germany } \\ \text { M. Melozzi } & \text { Italy } & \text { USA } \\ \text { R. Morris } & \text { Argentina } & \text { USA } \\ \text { T. Nagata } & \text { Japan } & \text { Australia } \\ \text { V. S. Niemela } & \text { Argentina } & \text { France } \\ \text { R. Nityananda } & \text { India } & \text { USA } \\ \text { K.-D. Oh } & \text { Korea } & \text { USA } \\ \text { A. R. Patnaik } & \text { India } & \text { USA } \\ \text { Y. Sobouti } & \text { Iran } & \text { USA } \\ \text { C. Sterken } & \text { Belgium } & \text { New Zealand } \\ \text { K. Subramanian } & \text { India } & \text { England } \\ \text { J. Trujillo } & \text { Germany } & \text { USA } \\ \text { R. J. Wainscoat } & \text { Australia } & \text { Netherlands } \\ \text { Y. Wu } & \text { China } & \text { USA }\end{array}$

Approximately half this number of applications could not be granted because they were not within the guidelines set for the Commission (see IAU Information Bulletin no. 50). Further, a number of preliminary inquiries were not followed up when copies of the guidelines were sent. In a few cases, prospective grantees received funds from other sources and then did not require Commission 38 support. A few applications are still under consideration.

Thanks are due to Prof. Edith Muller, Prof. J. Delhaye and Mrs. Vicki Leino for valuable help in preparing this report. 